\title{
Detection of auto antibodies and transplantation of cultured autologous melanocytes for the treatment of vitiligo
}

\author{
MEI-CAI ZHU ${ }^{1}$, HONG-YU MA ${ }^{1}$, ZHI ZHAN ${ }^{1}$, CHENG-GANG LIU $^{1}$, WEI LUO ${ }^{2}$ and GUANG ZHAO ${ }^{2}$ \\ ${ }^{1}$ Center of Clinical Laboratory; ${ }^{2}$ Department of Dermatology, Air Force General Hospital, Beijing 100036, P.R. China
}

Received April 25, 2015; Accepted July 13, 2016

DOI: $10.3892 /$ etm.2016.3949

\begin{abstract}
The aim of the present study was to establish an immunofluorescence method of antibody detection to identify melanocytes in the serum of vitiligo patients. Furthermore, we aimed to establish a method for the culture and proliferation of autologous pure melanocytes and to observe the effect of their transplantation for the treatment of vitiligo. Suspension of epidermal cells with melanocytes was performed using trypsin digestion of normal epiderm from eyelid operation and melanocytes were selectively cultured and proliferated in serum-free M2 medium. FITC-labeled rabbit anti-human antibody was used to detect the relative fluorescence intensity of the melanocytes. After identification with immunological and biological examinations, the melanocytes were transplanted to depigmented areas of vitiligo. Repigmentation was observed continuously. The results indicated that melanocytes could be selectively proliferated in the medium. Subsequently, pure melanocytes without contamination of fibroblast and keratinocyte were harvested. A total of 34 patients suffering vitiligo for between 3 months and 20 years with depigmented area (between $4 \mathrm{~cm}^{2}$ and $70 \%$ of body surface) were divided into 19 cases of developing stage and 15 cases of stable stage, according to the change of depigmentation. A total of 15 developing cases were positive for the antibody against melanocytes, with the positive rate of $79 \%$. The titers of serum was $>1: 50$ in 10 patients at the developing stage, and 5 developing patients were 1:10. Among the 15 stable cases, four were positive, with a positive rate of $27 \%$. Fluorescence of antibody was localized in the cytoplasm of the melanocytes. Autologous melanocytes of vitiligo patients could be selectively proliferated in the medium. Next, pure melanocytes without contamination with fibroblasts and keratinocytes were harvested. A total of 16 vitiligo patients with 28 depigmented areas $\left(2-200 \mathrm{~cm}^{2}\right)$ were treated with transplantation of melanocytes. Repigmentation
\end{abstract}

Correspondence to: Dr Mei-Cai Zhu, Center of Clinical Laboratory, Air Force General Hospital, 30 Fucheng Road, Beijing 100036, P.R. China

E-mail: zhumeicai_mcl@163.com

Key words: vitiligo, melanocyte culture, antibody against melanocytes, immunofluorescence, autologous transplantation of the transplanted areas appeared as red coloration after one month. All the vitiligous areas received transplantation were repigmented significantly with hypo- or hyper-pigmentation after 3-5 months. After 6-8 months, 87.5\% of lesions showed repigmentation of $>50 \%$ of the lesion area. No scarring or other side-effects occurred. After follow-up of 5 years, no relapse was observed in transplantation area. Thus, an immunofluorescence method for the test of antibody to melanocytes in the serum of vitiligo patients was established. Transplantation of cultured autologous melanocytes was an effective and safe measure for treatment of vitiligo, particularly for patients with a large depigmented area.

\section{Introduction}

Vitiligo is a common skin disease, with an incidence of $1-2 \%$ (1), in which melanocytes in the diseased area disappear, resulting in depigmentation. The pathomechanisms underlying vitiligo remain unclear, but may include: Autoimmune, defect in neural regulation, over oxidation caused by damage of reduction system, the recent emergence of the formation of inclusion bodies caused by defect in melanocyte absorption (2). It has been found that components of immune reactions could mediate melanocyte lysis in the serum of partial patients with vitiligo (3). IgG in the serum of patients with vitiligo could penetrate cultured melanocytes in vitro, causing apoptosis (4). These findings indicate that humoral immunity and autoantibodies play an important role in the occurrence and development of vitiligo. Establishing a detection method for anti-melanocyte antibodies, measuring antibody titers in the blood of patients with vitiligo and observing its association with the disease are crucial for understanding the pathogenesis of vitiligo, planning therapeutic interventions and assessing curative effects $(4,5)$. As determined by the present study, immunofluorescence testing of anti-melanocyte antibodies, melanocytes may be cultured in vitro and pure cells harvested. An advantage of this method is that there are sufficient uniform melanocytes for detecting the antibodies in patient samples. The main drawbacks are that the success rate of melanocyte culture is low, with a long cycle and high cost, and it is easy to develop contamination of skin fibroblasts and keratinocytes. On the basis of successfully cultured melanocytes, an immunofluorescence assay of anti-melanocyte antibodies may be conducted using the serum of vitiligo patients. The present study demonstrated that vitiligo patient serum may contain 
anti-melanocyte antibodies, and the antibody positive fluorescent coloration is located in the cytoplasm of melanocytes. The titer and the positive rate are associated with the progression and stability of disease. Certain drugs and physiotherapy, such as steroids and UVA, have poor efficacy for treating vitiligo. Autologous skin grafting to supplement melanocytes has been suggested to be an effective treatment for vitiligo $(6,7)$. However, it is difficult to treat cases involving large lesions due to the limited availability of graft skin. Sources of allogeneic melanocytes are more abundant; however, there may be a rejection reaction. There are only individual exploratory reports, with poor clinical results (8). Reports on transplantation of autologous melanocytes cultured in vitro to treat vitiligo have been published $(9,10)$. An advantage of this approach is that sufficient melanocytes can be harvested for a large area of the transplant. The main drawbacks are that the success rate of culture is low, with a long treatment cycle, and that a white border area between the transplant and the normal skin area may develop. Autologous melanocyte transplantation is suitable for patients in with stable phase vitiligo, while those in development stage may be vulnerable to relapse (11). In the present study, the levels of each patients' own immune fluorescent antibody were initially detected, and transplantation was used to treat the vitiligo patients in the stable stage with negative autoantibody and a smaller number of vitiligo patients in the development stage. The pigment of lesions was significantly recovered, without white border areas between the transplanted area and the normal skin. Moreover, there was no scarring or other notable side effects. No recurrence was observed within five years following transplantation. Thus, the present results indicate that in negative anti-melanocyte autoantibody patients, transplantation of cultured autologous melanocytes in vitro may be an effective, safe and suitable treatment for those with large skin lesions.

\section{Materials and methods}

Patient data. There were 36 patients (18 females and 18 males) enrolled in the study, aged between 6 and 43 years. The disease course ranged between 3 months and 20 years. The lesion in eight of the cases was confined to one area, and more than two areas of depigmentation were observed in 28 cases. Affected areas were varied, including the limbs, trunk, face and shank. The skin lesion area ranged from a minimum of $4 \mathrm{~cm}^{2}$ up to $70 \%$ of the body surface. If the depigmentation area did not expand and change, remaining stable for $>3$ years, it was identified as stable stage, and otherwise identified as development stage. Among the 36 cases, there were 20 cases in development stage and 16 cases in the stable stage. All patients had a history of drug or UVA treatment which was ineffective. Written informed consent was obtained from all patients. From each patient a 4-ml venous blood sample was harvested, which stood for $2 \mathrm{~h}$ at room temperature, followed by centrifugation at $4,000 \times \mathrm{g}$ for $10 \mathrm{~min}$. The serum was then placed in a blocking Eppendoff tube and preserved at $-20^{\circ} \mathrm{C}$. Normal serum was also collected from 36 healthy subjects (18 males and 18 females) who were aged 6-43 years.

Reagents. M2 melanocyte growth medium (DMEM/F12, containing $5 \mathrm{ng} / \mathrm{ml}$ basic fibroblast growth factor); anti-melanocyte tyrosinase-related protein 75 (TRP-75) monoclonal antibody (cat no. SAB2102617), S-100 monoclonal antibody (cat no. S2657), mouse anti-human fibroblasts and keratinocytes monoclonal antibody (cat no. C6909); FITC-labeled goat anti-mouse IgG (cat no. F9006). and L-DOPA were purchased from Sigma-Aldrich.

Preparation of epidermal cell suspensions. Normal eyelid skin was collected after surgery, scissors were used to cut the epidermis, then samples were placed in saline and digested with $0.25 \%$ trypsin at $37^{\circ} \mathrm{C}$ for $15 \mathrm{~min}$ or at $4^{\circ} \mathrm{C}$ overnight. Samples were subsequently centrifuged at 4,000 x $\mathrm{g}$ for $10 \mathrm{~min}$, then precipitate was collected and resuspended in culture medium.

Melanocyte culture. According to previously described methods $(11,12)$, the epidermal melanocytes were suspended in M2 (DMEM/F12 medium containing $5 \mathrm{ng} / \mathrm{ml}$ recombinant human basic fibroblast growth factor; bFGF) melanocyte selective medium, which was changed once every three days. After melanocytes fused, they were digested with trypsin from culture flask. After the cells were cultured for 4 or 5 passages, melanocytes were digested with trypsin from the culture flask, centrifuged for $10 \mathrm{~min}$ at 4,000 x $\mathrm{g}$. The precipitate was collected, resuspended in culture medium, and stored for subsequent experiments.

Melanocyte identification. The morphology of the cultured cells was observed using an inverted microscope. TRP-75 fluorescent immunostaining was performed as previously described (13). Briefly, cells were seeded on glass slides treated with gelatin. When the fusion rate reached $70 \%$, the cells were washed twice with phosphate-buffered saline (PBS), fixed with cold acetone, added anti-TRP-75 monoclonal antibody at room temperature for $1 \mathrm{~h}$, added FITC-labeled goat anti-mouse secondary antibody at room temperature $1 \mathrm{~h}$. After staining with dopamine, cells were fixed with cold methanol, put into $0.1 \%$ L-DOPA phosphate buffer $(\mathrm{pH} 7.4)$ and incubated at $37^{\circ} \mathrm{C}$, then observed for the degree of coloration compared with normal skin.

Immunofluorescent staining of anti-melanocyte antibody. After the slides were washed with distilled water for $10 \mathrm{~min}$ and autoclaved $120^{\circ} \mathrm{C}$ for $30 \mathrm{~min}$, coated with $0.2 \%$ gelatin for $30 \mathrm{~min}$ and liquid was removed using a pipette. The slides were fixed with $0.5 \%$ formaldehyde for $30 \mathrm{~min}$, washed three times with PBS (5 min each time), dried and set aside. A monolayer of melanocytes in a culture flask was digested with trypsin and counted with a hemocytometer by microscope, used culture medium to prepare $1 \times 10^{5} / \mathrm{ml}$ cell suspension. The slides were marked with 3 or 4 circles with a marker, and a 100-200- $\mu$ l cell suspension was inoculated into each circle, put in wet box and cultured in $5 \% \mathrm{CO}_{2}$ at $37^{\circ} \mathrm{C}$. When the fusion rate reached $70 \%$, after the removal of liquid, the specimens were washed three times with PBS ( 3 min each time), fixed with cold acetone, washed three times with PBS, dried and set aside. Then, $10 \%$ rabbit serum was used to seal the cells on glass slides for $60 \mathrm{~min}$, which were then washed three times with PBS (3 min each time). The patient or normal human serum, after 1:10, 1:50 and 1:100 proportional dilution, was 
added at a volume of $100 \mu 1$ per well, PBS was added to a well as a negative control, incubated for $1 \mathrm{~h}$ at room temperature and washed three times with PBS (3 min each time). Next, FITC-labeled rabbit anti-human IgG antibody (1:50) was added for $1 \mathrm{~h}$ at room temperature, then washed three times with PBS (3 min each time). Samples were blotted up PBS, sealed with mounting medium and observed under a fluorescence microscope. When serum was diluted to 1:10, melanocytes that had immune fluorescence and intensity higher than the PBS negative control were considered to be positive.

Evaluation of safety for tumorigenesis and mycoplasma detection. Approximately $10^{4}$ melanocytes were suspended in $0.1 \mathrm{ml}$ saline and injected (intracutaneously) into nude mice. Subsequently, $10^{5}$ melanocytes were suspended in $0.2 \mathrm{ml}$ saline and injected (subcutaneously) into the nude mice. Each mouse was injected with two parts, a total of six mice. The injection site was consecutively observed after three months, dissected and the injection site and pathological morphology of major organs were observed. Mycoplasma detection was conducted using Hoechst 33258 staining.

Cultured autologous melanocytes of vitiligo patients. Suction was applied to induce a blister using an epidermal separator on the normal skin area of vitiligo patients. Working pressure was $48 \mathrm{kPa}$, at $43^{\circ} \mathrm{C}$ for $1 \mathrm{~h}$, using scissors to cut the skin when blisters formed. Then, samples were placed in saline, digested with $0.25 \%$ trypsin at $37^{\circ} \mathrm{C}$ for 15 min or $4^{\circ} \mathrm{C}$ overnight. Samples were centrifuged at $4,000 \mathrm{x} \mathrm{g}$ for $10 \mathrm{~min}$, the pellet was collected then resuspended in culture medium. The epidermal melanocytes were suspended in M2 (DMEM/F12 medium containing $5 \mathrm{ng} / \mathrm{ml}$ human recombinant bFGF) melanocyte selective medium. Medium was changed once every three days, when melanocytes fused they were digested with trypsin for subculture, as described in previous studies $(11,12)$. After subculture for 4 or 5 generations, digested melanocytes with trypsin from flasks, and the melanoma cells were collected by centrifugation at $4,000 \mathrm{x}$ g for $10 \mathrm{~min}$, then suspended in medium. The concentration of cells was $7 \times 10^{4}$ melanocytes $/ \mathrm{ml}$.

Cell transplantation. The lesion area was sterilized. When the skin area was larger, a surface anesthetic was applied, when the area was smaller, used injection anesthesia was employed. The lesions were gently wiped with a scalpel to avoid scarring, melanocytes suspended in a small amount of medium were transferred to the transplant area with $7,000 \mathrm{cell} / \mathrm{cm}^{2}$, evenly coated over the skin surface using a sterile glass rod. The lesion area was covered with layer of Vaseline gauze, and covered with sterile gauze, let stand for $1 \mathrm{~h}$.

\section{Results}

Cell culture. After epidermal cells were seeded in culture bottles for $4 \mathrm{~h}$, they began to exhibit adherence. After 2-3 days, melanocytes were stretched in length, pseudopodia and branches appeared. After seven days, the cells showed typical morphology of melanocytes, primarily fusiform bipolar type cells with branches (Fig. 1). After 4 or 5 generations ( 40 days) cultured generations reached the required transplantation quantity of $7,000 \mathrm{cell} / \mathrm{cm}^{2}$. Anti-TRP-75 fluorescence staining

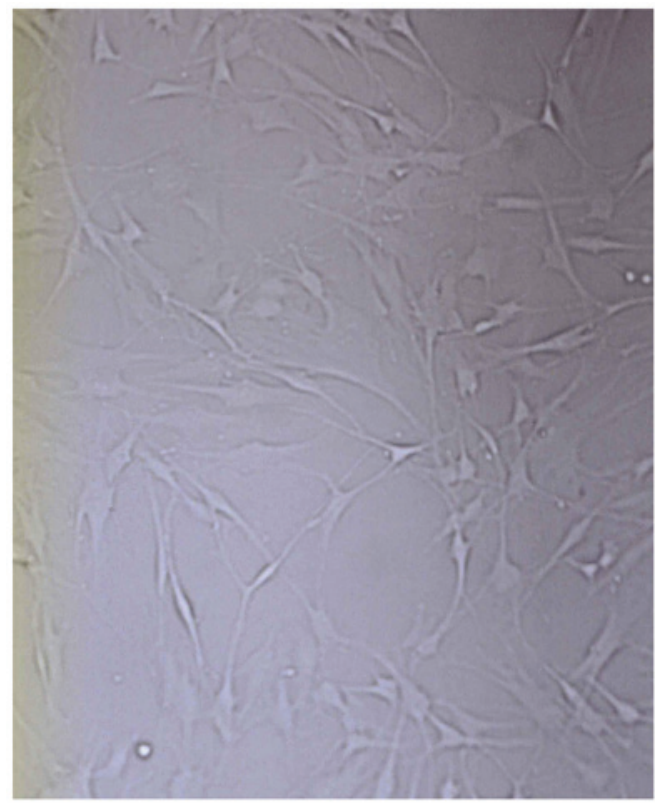

Figure 1. Morphology of cultured melanocytes in vitro (magnification, $\mathrm{x} 400$ ).

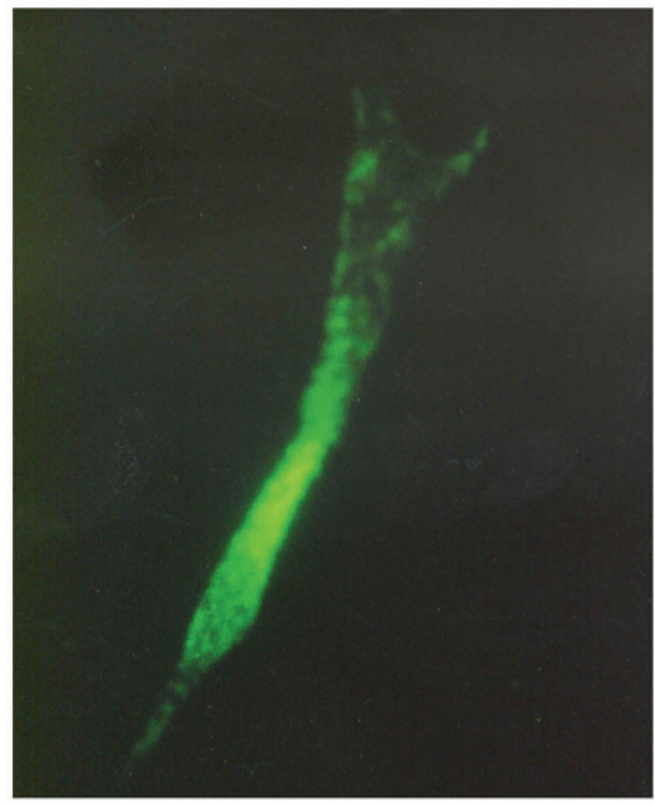

Figure 2. Trp75 fluorescence immune staining of cultured melanocytes in vitro (magnification, $\mathrm{x} 1,000)$.

was used to display cytoplasmic morphology at high magnification (Fig. 2). Anti-fibroblast and keratinocyte-specific antigen staining resulted in no coloration, indicating that there was no contamination of both types of the cells. Dopamine cytoplasmic staining showed as brown or dark gray (Fig. 3). The mycoplasma of cell cultures exhibited no contamination. Subsequently, $10^{4}$ - and $10^{5}$-melanocyte suspensions were injected into nude mice. The mice were continuously observed for three months, at which point the injection sites showed no lumps. After dissection, injection sites and organs showed no pathological changes.

Detection of anti-melanocyte antibodies of vitiligo serum. A total of 36 cases of vitiligo disease and immunofluorescent titer 
Table I. Feature of skin area and effect of autologous melanocytes transplantation of 16 cases of vitiligo.

\begin{tabular}{rlclccc}
\hline No. & Gender & Age (years) & \multicolumn{1}{c}{ Sites } & Skin lesions (n) & Size $\left(\mathrm{cm}^{2}\right)$ & Degree of recovery \\
\hline 1 & Male & 28 & Leg & 1 & 16 & Good \\
2 & Female & 19 & Leg & 2 & 15 & Good \\
3 & Male & 25 & Face & 1 & 12 & Good \\
4 & Male & 29 & Shoulder, ear & 3 & 9 & Good \\
5 & Male & 20 & Face & 4 & 200 & Good \\
6 & Female & 21 & Neck & 1 & 9 & Bad \\
7 & Female & 18 & Chest, neck & 3 & 18 & Good \\
8 & Female & 16 & Leg & 1 & 11 & Bood \\
9 & Male & 34 & Eyelid & 1 & 4 & Good \\
10 & Female & 41 & Face & 1 & 2 & Medium \\
11 & Female & 16 & Neck & 1 & 3 & Medium \\
12 & Male & 21 & Forehead & 3 & 23 & Good \\
13 & Female & 24 & Forehead, eyelid & 2 & 8 & Medium \\
14 & Female & 25 & Leg & 4 & 50 & Medium \\
15 & Male & 21 & Neck & 1 & 11 & 18 \\
16 & Female & 40 & Neck & 1 & & \\
\hline
\end{tabular}

Good, repigmentation area accounted for $>80 \%$; Medium, repigmentation area accounted for 50-80\%; Bad, repigmentation area accounted for $<50 \%$.

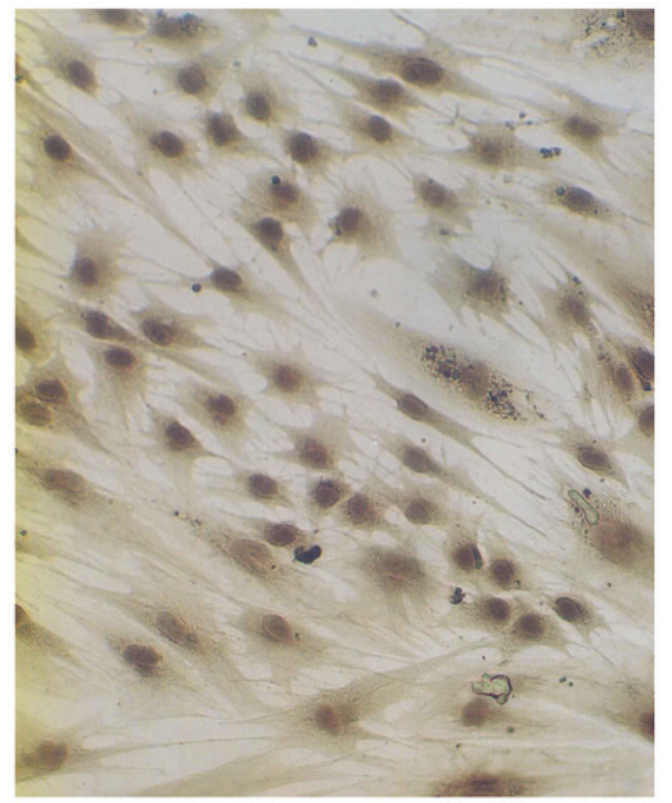

Figure 3. Dopamine staining of cultured melanocytes in vitro (magnification, x400).

of anti-melanocyte antibody in serum are shown in Table I. A total of 20 cases of patients' immunofluorescent titer in serum antibody were $>1: 10$ among 30 cases, the total positive rate was $56 \%$. Furthermore, 16 cases were antibody positive among 20 cases of anti-melanocyte patients in the development stage, the antibody positive rate was $80 \%$; 11 cases of which were $>1: 50$ and five cases of antibody titer were 1:10. Four cases were antibody positive among 16 cases of anti-melanocyte patients in stable stage, and the antibody positive rate was $25 \%$. A single case of antibody titer was 1:50. Three cases of antibody titer were 1:10 fluorescence of antibody positive located in the cytoplasm of melanocytes. (Fig. 4A and B). Normal serum controls were collected from 36 healthy subjects (18 males and 18 females) who were aged 6-43 years, which were diluted in 1:10. The immunofluorescence intensity of melanocytes was no higher than the PBS-treated negative control.

Transplantation effect. The serum was diluted by 1:10 in 16 cases with negative serum anti-melanocyte antibody, including 12 cases in stable stage and four cases in development stage, which accepted transplantation of autologous melanocytes (Table I). After 7-10 days, wound healing was observed at the transplantation site. After 30 days, the transplantation site turned red and slight pigmentation appeared. At the edge of transplantation, there was a white dividing line with shallow junction of normal skin. At 3-5 months after transplantation, the pigmentation was deepened and the border disappeared compared with the surrounding normal skin. After 6-8 months, pigmentation was stably restored, and the skin color was similar to that of the adjacent normal skin (Fig. 5A-D). The normal skin and transplantation area did not have white borders and scar as well as other side effects. At two months after transplantation, pigment was restored to near normal levels in certain cases. A total of 16 patients had 28 skin whitened areas, including seven males and nine females, aged 19-40 years.

Four cases at legs, seven cases at the forehead and face (two cases at the eyelid), four cases at the neck, one case at the shoulder and ears, with an area of $2-200 \mathrm{~cm}^{2}$. The repigmentation area accounted for $>80,50-80$ and $<50 \%$ of the transplant area in nine, five and two cases, respectively. Patients with a repigmentation area of $>50 \%$ accounted for $87.5 \%$ among all patients with transplantation. The lower 
A

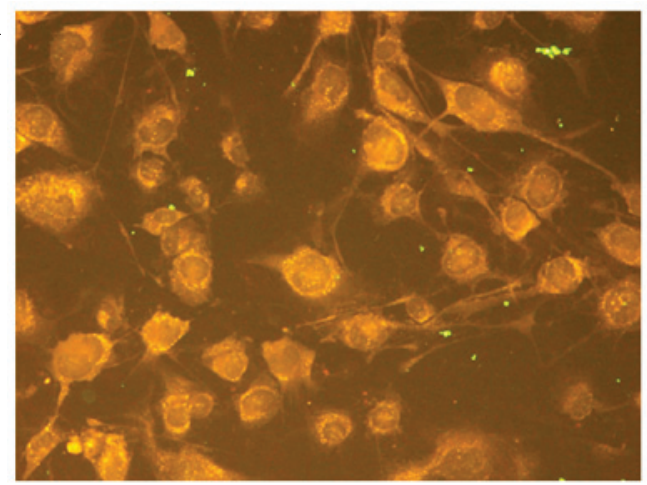

B

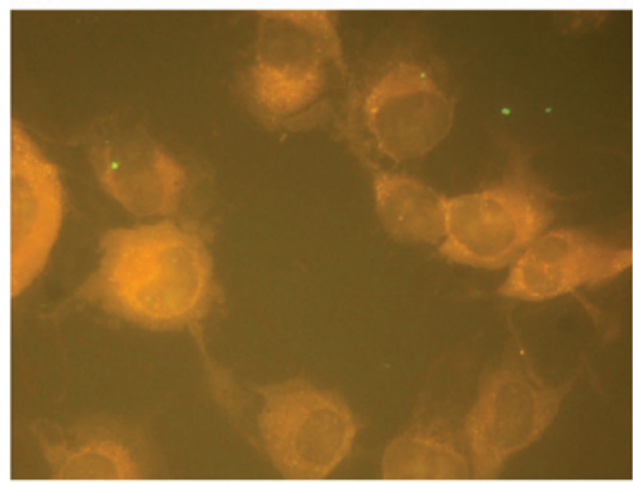

Figure 4. Immunofluorescence staining of anti-melanocyte in serum of vitiligo (1:50 dilution) at (A) x400 and (B) x1,000 magnification.
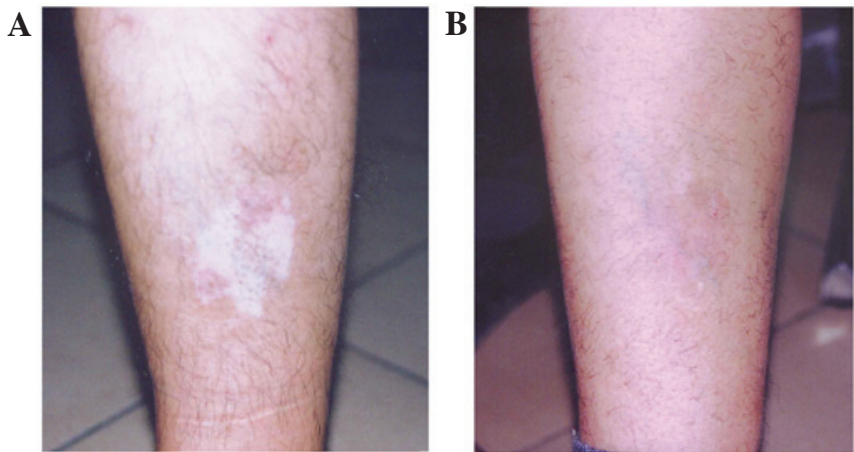

C

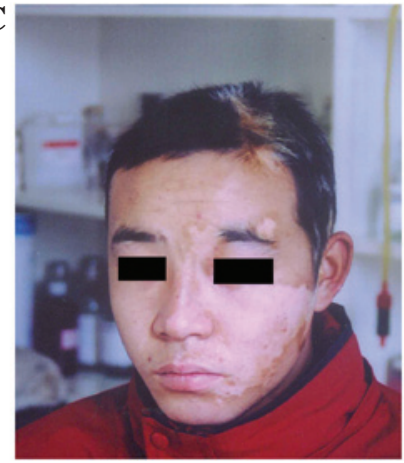

D

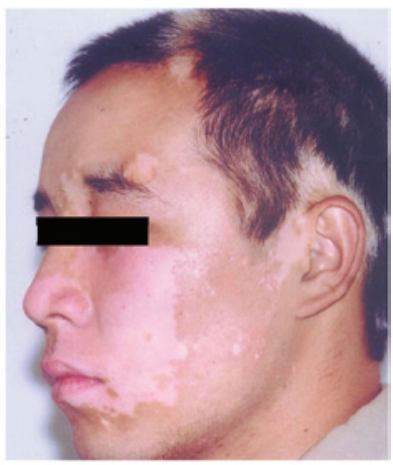

Figure 5. Effect of autologous transplantation of cultured melanocytes in vitro. Patient leg (A) before transplantation and at (B) 6 months after transplantation. Patient face (C) before transplantation and at (D) 2 months after transplantation.

limbs, torso and face were colored evenly and the effect was promising (repigmentation area accounted for $>80 \%$ ). Neck, eyelids, lips and other sites were sometimes unevenly colored; however, reservoirs of pigmentation were formed. In exposed parts of the transplanted area, it was colored faster. The transplantation area was observed at five years following transplantation, and no recurrence was observed.

\section{Discussion}

Vitiligo is characterized by the disappearance of pigment cells. Due to unclear factors, melanocytes of skin lesions disappear, and the incidence showed an increasing trend in recent years (1). The aim of the present study was to investigate the pathogenesis of vitiligo, which is important for developing novel clinical treatments. Lymphocyte infiltration was observed at the edge of vitiligo lesion area, and anti-melanocyte antibody was detected in the blood, which indicated that abnormal autoimmunity was one of the underlying causes of vitiligo (2). In addition, the present results showed an increased percentage and high levels of anti-melanocyte antibodies in the serum of patients with vitiligo in the development phase, suggesting that humoral immunological mechanisms played an important role in the occurrence and development of vitiligo. The amplification of autologous melanocytes cultured in vitro was performed to obtain sufficient numbers of pure melanocytes, providing the necessary conditions for the establishment of immunofluorescent antibody detection. The immunofluorescence of melanocyte antibody was established, was simple to perform and easy to observe, laying the foundation for the development of clinical testing. Fluorescence detection was used for antibody located in the cytoplasm of melanocytes, indicating that melanocyte autoantigens were located in the cytoplasm. Melanocyte-specific protein tyrosinase, TRP-1,TRP-2 and melanin-concentrating hormone receptor 1 , which may be autoantigen components $(14,15)$.

Drugs and physiotherapy do not show good efficacy for vitiligo patients (7). The source of allogeneic melanocytes is more convenient; however, there may be a rejection reaction. There are only individual exploratory reports, with poor clinical results (8). Transplantation of autologous melanocytes is an effective therapeutic method. There are several approaches for transplantation of autologous melanocytes: Direct transplantation of autologous skin (6), autologous epidermal cell suspension (16) and autologous melanoma cells are cultured in vitro. Melanocytes only account for $2-3 \%$ of skin cells. As melanocytes are too few in autologous epidermal cell suspension, they are not suitable for large-scale migration (12). By amplification of autologous melanocytes cultured in vitro, sufficient numbers of pure melanocytes may be harvested for transplantation, as determined by the present study. Previous studies observed no rejection reaction after transplantation, with stable effects. When melanocytes were cultured, fibroblasts and keratinocytes account for a high proportion and had fast growth. Thus, it was necessary to inhibit fibroblasts and keratinocyte cells and make selective growth of melanocytes $(17,18)$. In order to continuously subculture melanocytes in vitro, tissue plasminogen activator (TPA), CT, IMBX and bovine pituitary extract have been added in early medium to promote cell division or growth agents (19). TPA is a potent 
mitotic accelerator, and the carcinogenic risk is possible, CT is toxic and bovine pituitary extract has the risk of spreading diseases like cerebral cavernous malformation. (11) For these reasons, the above ingredients were used to culture melanocytes which were not suitable for clinical treatment in the present study. The medium was used to inhibit the growth of fibroblasts and keratinocytes. Melanocytes had no contamination by these two cells for 4-5 passages. Furthermore, $5 \mathrm{ng} / \mathrm{ml}$ bFGF functioned as a melanocyte growth promoter, which is the normal growth factor in the body and is able to eliminate or minimize the risk factors (11). The medium was used to culture melanocytes in vitro for 20 passages, and the cells maintained good growth. A total of 28 lesions among 16 patients were treated by the transplantation of melanocytes. The legs, trunk and face were evenly colored, and the effect was better. The neck, eyelids, lips and other sites were unevenly colored. This result may be related to the site which was in active stage against uniform growth of transplanted cells. In exposed parts of the transplanted area colored faster, it was indicated that sunlight can promote pigment-production of transplanted cells. The stable stage and development stage of positive and negative patients were detected by immunofluorescence testing of melanocyte antibody, which was important for preventing and reducing recurrence after transplantation of melanocytes.

\section{Acknowledgements}

The current study would like to thank Ms. Wang Hong for her assistance in the manuscript.

\section{References}

1. Lu T, Gao TT, Wang AH, Li Q, Li CY, Zhao XD and Sun DJ: The prevalence of vitiligo in Shaanxi Province of China. Zhong Hua Pi Fu Ke Za Zhi 37: 406-407, 2004 (In Chinese).

2. Namazi MR: Neurogenic dysregulation, oxdative stress, autoimmunity and melanocytorrhagy in vitiligo: Can they be interconnected? Pigment Cell Res 20: 360-363, 2007.

3. Cui J, Arita Y and Bystryn JC: Cytolytic antibodies to melanocytes in vitiligo. J Invest Dermatol 100: 812-825, 1993.

4. Ruiz-Argüelles A, Brito GJ, Reyes-Izquierdo P, Pérez-Romano B and Sánchez-Sosa S: Apoptosis of melanocytes in vitiligo results from antibody penetration. J Autoimmun 29: 281-286, 2007.
5. Sandoval-Cruz M, García-Carrasco M, Sánchez-Porras R, Mendoza-Pinto C, Jiménez-Hernández M, Munguía-Realpozo $\mathrm{P}$ and Ruiz-Argüelles A: Immunopathogenesis of vitiligo. Autoimmun Rev 10: 762-765, 2011.

6. Horikawa T, Mishima Y, Nishino K and Ichihashi M: Horizontal and vertical pigment spread into surrounding piebald epidermis and hair follicles after suction blister epidermal grafting. Pigment Cell Res 12: 175-180, 1999.

7. Boersma BR, Westerhof W and Bos JD: Repigmentation in vitiligo vulgaris by autologous minigrafting: Results in nineteen patients. J Am Acad Dermatol 33: 990-995, 1995.

8. Lu T, Gao TW, Liu YF, Li CY and Sun LC: The explore of allogenic transplantation of melanocytes to treat vitiligo. Zhong Guo Pi Pu Xing Bing Xue Za Zhi 15: 240-242, 2001 (In Chinese).

9. Mulekar SV: Melanocyte-keratinocyte cell transplantation for stable vitiligo. Int J Dermatol 42: 132-136, 2003.

10. Chen YF, Chang JS, Yang PY, Hung CM, Huang MH and Hu DN: Transplant of cultured autologous pure melanocytes after laser-abrasion for the treatment of segmental vitiligo. J Dermatol 27: 434-439, 2000.

11. Olsson MJ and Juhlin L: Long-term follow-up of leucoderma patients treated with transplants of autologous cultured melanocytes, ultrathin epidermal sheets and basal cell layer suspension. Br J Dermatol 147: 893-904, 2002.

12. Olsson MJ and Juhlin L: Leucoderma treated by transplantation of a basal cell layer enriched suspension. Br J Dermatol 138: 644-648, 1998.

13. Beck AJ, Phillips J, Smith-Thomas L, Short RD and MacNeil S: Development of a plasma-polymerized surface suitable for the transplantation of keratinocyte-melanocyte cocultures for patients with vitiligo. Tissue Eng 9: 1123-1131, 2003.

14. Le-Poole IC and Luiten RM: Autoimmune etiology of generalized vitiligo. Curr Dir Autoimmun 10: 227-243, 2008

15. Kemp EH, Waterman EA, Hawes BE, O'Neill K, Gottumukkala RV, Gawkrodger DJ, Weetman AP and Watson PF: The melanin-concentrating hormone receptor 1 , a novel target of autoantibody responses in vitiligo. J Clin Invest 109: 923-930, 2002.

16. Kao CH, Ko WC, Ko SS and Tsai RY: A comparative study on autologous graft for segmental type vitiligo. Dermatol Sinica 13: 65-74, 1995.

17. Halaban R and Alfano FD: Selective elimination of fibroblasts from cultures of normal human melanocytes. In Vitro 20: 447-450, 1984.

18. Phillips J, Gawkrodger DJ, Caddy CM, Hedley S, Dawson RA, Smith-Thomas L, Freedlander E and Mac Neil S: Keratinocytes suppress TRP-1 expression and reduce cell number of co-cultured melanocytes: Implications for grafting of patients with vitiligo. Pigment Cell Res 14: 116-125, 2001.

19. Eisinger M and Mark O: Selective proliferation of normal human melanocytes in the presence of phorbol ester and cholera toxin. Proc Natl Acad Sci USA 79: 2018-2022, 1982. 